\title{
Evaluating cutaneous photoaging by use of multiphoton fluorescence and second-harmonic generation microscopy
}

\author{
Sung-Jan Lin \\ Dermatology, Taipei Campus and Yun-Lin Branch, National Taiwan University Hospital, Taiwan, and Institute of \\ Biomedical Engineering, National Taiwan University, Taipei, Taiwan \\ Ruei-Jr Wu \\ Department of Physics, National Taiwan University, Taipei, Taiwan
}

Hsin-Yuan Tan

Institute of Biomedical Engineering, National Taiwan University, Taipei, Taiwan, and Department of Ophthalmology, Chang Gung Memorial Hospital, Linko, Taiwan

Wen Lo

Department of Physics, National Taiwan University, Taipei, Taiwan

Wei-Chou Lin

Department of Pathology, National Taiwan University Hospital, Taipei, Taiwan

Tai-Horng Young

Institute of Biomedical Engineering, National Taiwan University, Taipei, Taiwan

Chih-Jung Hsu, Jau-Shiuh Chen, and Shiou-Hwa Jee

Department of Dermatology, National Taiwan University Hospital and National Taiwan University College of Medicine, Taipei, Taiwan

Chen-Yuan Dong

Department of Physics, National Taiwan University, Taipei, Taiwan

Received March 9, 2005; revised manuscript received April 20, 2005; accepted April 24, 2005

The photoaging process of facial skin is investigated by use of multiphoton fluorescence and secondharmonic generation (SHG) microscopy. We obtain the autofluorescence (AF) and SHG images of the superficial dermis from the facial skin of three patients aged 20,40, and 70 years. The results show that areas of $\mathrm{AF}$ increase with age, whereas areas of SHG decrease with age. The results are consistent with the histological findings in which collagen is progressively replaced by elastic fibers. The AF and SHG changes in photoaging are quantified by a SHG to autofluorescence aging index of dermis (SAAID). Our results suggest that SAAID can be a good indicator of the severity of photoaging. () 2005 Optical Society of America

OCIS codes: $190.4160,170.3880,180.0180$.

Cutaneous photoaging is caused by cumulative damage from sunlight exposure. ${ }^{1}$ The ultraviolet spectrum of sunlight is believed to play a major role in causing cutaneous photoaging. Dryness, irregular pigmentation, and wrinkling are the clinical features of photoaging. Histologically, photoaging can be evaluated by changes in the extracellular matrix components of the superficial dermis. ${ }^{1}$ In cases of severe photoaging, increased disorganized elastic fibers can be demonstrated in the superficial dermis (solar elastosis). A number of surgical procedures and topical medication are used to rejuvenate photoaged skin aimed at remodeling collagen and elastic fibers, including laser resurfacing, ${ }^{2}$ intense pulse light treatment, ${ }^{3}$ chemical peeling, ${ }^{4}$ and topical tretinoids. ${ }^{1}$ Evaluation of the treatment response usually depends on subjective clinical assessment by physicians and patients because of lack of a reliable noninvasive quantification method.

Recently, multiphoton fluorescence (MPF) microscopy has gained much popularity in biomedical imaging. ${ }^{5,6}$ The nonlinear excitation of fluorescence photons by use of ultrafast, near-infrared excitation sources has important advantages in its ability to acquire enhanced axial depth discrimination images, reduced overall specimen photodamage, and increased imaging penetration depths compared with single-photon confocal imaging. In addition to morphological information, characteristic autofluorescence (AF) from various cells and luminescence from components of the extracellular matrix can help specifically feature subjects of interest. ${ }^{7}$ Compared with 
collagen fibers, elastic fibers are more effective in generating $\mathrm{AF}$ when a longer excitation wavelength is used. ${ }^{8}$ In addition to AF, noncentrosymmetric molecules including collagen, muscle fibers, and microtubules, can contribute to a second-harmonic generation (SHG) signal. ${ }^{7,9-11}$ Collagen is a particularly interesting subject for SHG imaging because of its wide distribution in various tissues, including skin. Due to its special molecular structures, we have shown that SHG imaging can be used to monitor the heat-induced structural transitions of collagen. ${ }^{12}$ In this work, we combine AF and SHG images to study photoaging. In comparison with histological results, our results show that $\mathrm{AF}$ can be used to quantify the elastic fibers and SHG can be used to quantify the collagen components of skin. We propose a SHG to autofluorescence aging index of dermis (SAAID) to quantify skin photoaging.

The MPF and SHG microscopic system used in this study is a modified version of a home-built laser scanning microscopic imaging system based on an upright microscope (E800, Nikon, Japan) described previously. ${ }^{13}$ A diode-pumped (Millennia X, SpectraPhysics, Mountain View, California) Ti:sapphire laser (ti-sa, Tsunami, Spectra-Physics) is used as the excitation source. The $760 \mathrm{~nm}$ output of the ti-sa laser is scanned in the focal plane by a galvanometer-driver $x-y$ mirror scanning system (Model 6220, Cambridge Technology, Cambridge, Massachusetts). For highresolution imaging, a high-numerical-aperture, oilimmersion objective (S Fluor $40 \times$, N.A. 1.3, Nikon) is used. A short-pass dichroic mirror (700DCSPRUV, Chroma Technology, Brattleboro, Vermont) is used to reflect the incident excitation laser source to the sample. The average laser power at the sample is $5.5 \mathrm{~mW}$. The generated AF and SHG signals are collected by the same objective. Before reaching the photodetectors, the AF and SHG signals are separated by a secondary dichroic mirror (435DCSX, Chroma Technology). The SHG signal centered at $380 \mathrm{~nm}$ is reflected by the secondary dichroic mirror and further filtered by a bandpass filter (HQ380/20, Chroma Technology), whereas the longer-wavelength AF passes through the dichroic mirror and a broadbandpass filter (E435LP, Chroma Technology) before being detected. The signal photons are processed by a single-photon counting photomultiplier tube (R7400P, Hamamatsu, Japan) and a home-built discriminator.

The facial skin specimens were obtained from the cheeks of three patients aged 20,40 , and 70 years undergoing facial surgery. A thin cross-sectional slice of formalin-fixed samples was mounted on the slide and covered with a No. 1.5 coverslip. Large-area multiphoton imaging was performed for each specimen, and a representative image subsection composed of a 3 by 3 image array along the epidermal-dermal junction is shown in Fig. 1. Since photoaging is most prevalent in the superficial dermal layers just below the epidermal-dermal junction, we randomly selected five $100 \mu \mathrm{m}$ by $200 \mu \mathrm{m}$ rectangular areas in this region for quantitative analysis. In each rectangular area, the pixels of SHG are defined as $a$ and

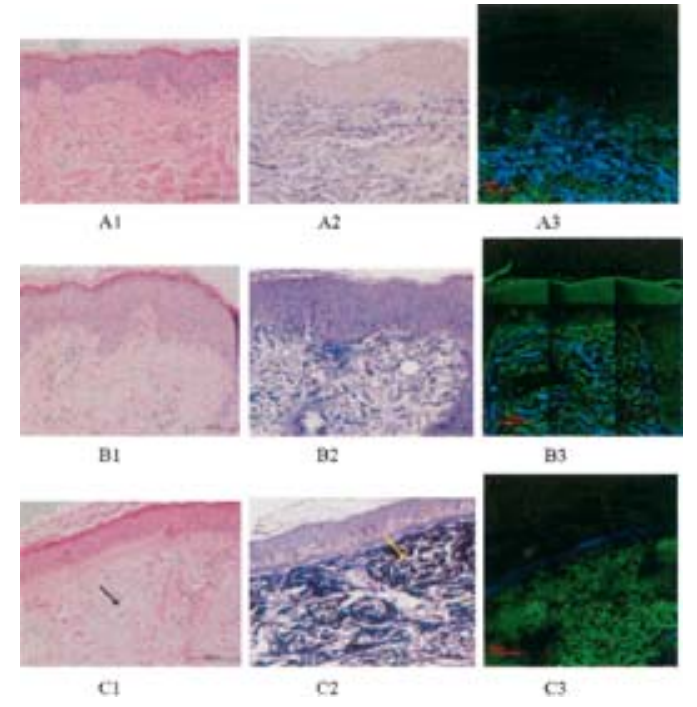

Fig. 1. H\&E stains (A1, B1, C1), elastic stains (A2, B2, C2), and AF and SHG images (A3, B3, C3; SHG signals are blue and AF signals are green) of the three cases ( 20-year-old, A1-A3; 40-year-old, B1-B3; 70-year-old, C1C3). Bars, $100 \mu \mathrm{m}$ in H\&E stains and elastic stains, $50 \mu \mathrm{m}$ in $\mathrm{AF}$ and $\mathrm{SHG}$ images.

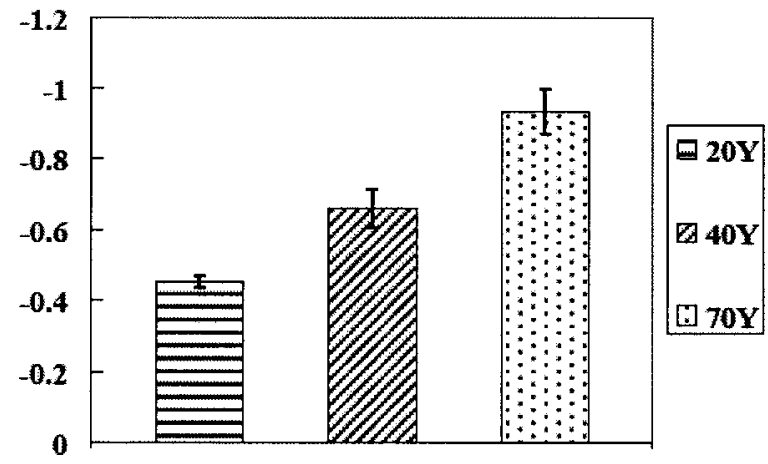

Fig. 2. SAAID of the three cases. Error bars represent the standard deviations calculated from SAAID determinations from five randomly chosen areas in the superficial dermis.

pixels of AF are defined as $b$. The SAAID is defined as $(a-b) /(a+b)$. In this nomenclature, SAAID decreases with photoaging, approaching -1 when collagen is completely replaced by elastic fibers. The index of each rectangular area is computed, and the average index in each case is calculated. For comparison, the specimens are further processed for histological examination with hematoxylin and eosin $(\mathrm{H} \& \mathrm{E})$ stains and elastic stains (Verhoeff-van Gieson stain). In H\&E stains, elastin and collagen have varying degrees of pink staining and solar elastosis is characterized by bluish gray curvy fibers in the superficial dermis. In elastic stains, elastic fibers are stained blue black and collagen fibers are red.

The H\&E stains, elastic stains, and AF and SHG images of the three cases are shown in Fig. 1 and the SAAIDs of the three cases are shown in Fig. 2. Judging from the $\mathrm{AF}$ and $\mathrm{SHG}$ images, several interesting results are obtained. With aging, areas of AF increase and SHG signals decrease in the superficial dermis. 
In the 20-year-old case, SHG signals and AF signals are evenly mixed. In the 40 -year-old case, proportions of AF signals increase in the dermis and SHG signals decrease. In the 70-year-old case, the dermis is almost completely composed of $\mathrm{AF}$ signals, and scanty SHG signals can only be demonstrated in the superficial dermis right below the epidermal-dermal junction.

A similar trend of the changes in the proportion of elastic tissue and collagen fibers is revealed in the histological findings. In $\mathrm{H} \& \mathrm{E}$ stains, elastic tissue cannot be clearly differentiated in the cases of the 20and 40-year-olds. The elastic stains show a slight increase in elastic tissue in the case of the 40-year-old. In the case of the 70-year-old, solar elastosis characterized by the presence of curvy bluish fibers is observed in H\&E staining (black arrow). This observation is further confirmed by the elastic stains in which a large amount of black elastic tissue is present in the superficial dermis (yellow arrow). The areas of elastic tissue in the histology are consistent within the areas of AF in the AF and SHG images. A negligible change in the $\mathrm{AF}$ associated with elastic fibers in fresh and formalin-fixed specimens has been demonstrated. ${ }^{8}$ Hence, in our setting, AF can be used to represent the elastic tissue, whereas SHG signals reflect collagen in the dermis.

To better quantify the changes of elastic and collagen fibers, the SAAID is shown in Fig. 2. Consistent with the above-mentioned findings, the SAAID decreases with age, showing the trend of an increasing elastic component of the dermis with photoaging. In the case of the 70-year-old, the average SAAID is -0.93 , indicating that the collagen fibers are almost completely replaced by elastic fibers.

In conclusion, MPF and SHG microscopy can be used to evaluate cutaneous photoaging. AF signals feature elastic fibers and SHG signals reflect collagen fibers in the dermis. Solar elastosis can be clearly demonstrated by the presence of masses of autofluorescent materials in the superficial dermis. The index SAAID can be used to quantify photoaging. Our results suggest that MPF and SHG microscopy can be further developed into noninvasive imaging tools for the clinical evaluation of photoaging.

S. H. Jee's e-mail address is shiouhwa@ha.mc.ntu.edu.tw; C. Y. Dong's is cydong@phys.ntu.edu.tw.

\section{References}

1. M. Yarr and B. Gilchrest, in Fitzpatrick's Dermatology in General Medicine, 6th ed. (McGraw-Hill, 2003), pp. 1386-1398.

2. W. Manuskiatti, R. Fitzpatrick, and M. Goldman, J. Am. Acad. Dermatol. 40, 401 (1999).

3. D. Kligman and Y. Zhen, Dermatol. Surg. 30, 1085 (2004).

4. P. Butler, S. Gonzalez, M. Randolph, J. Kim, N. Kollias, and M. Yaremchuk, Plast. Reconstr. Surg. 107, 222 (2001).

5. W. Denk, J. Strickler, and W. Webb, Science 248, 73 (1990).

6. P. So, C. Dong, B. Masters, and K. Berland, Annu. Rev. Biomed. Eng. 2, 399 (2000).

7. W. Zipfel, R. Williams, R. Christie, A. Nikitin, B. Hyman, and W. Webb, Proc. Natl. Acad. Sci. U.S.A. 100, 7075 (2003).

8. A. Zoumi, X. Lu, G. Kassab, and B. Tromberg, Biophys. J. 87, 2778 (2004).

9. A. Zoumi, A. Yeh, and B. Tromberg, Proc. Natl. Acad. Sci. U.S.A. 99, 11014 (2002).

10. P. Campagnola and L. Loew, Nat. Biotechnol. 21, 1356 (2003).

11. E. Brown, T. McKee, E. diTomaso, A. Pluen, B. Seed, Y. Boucher, and R. Jain, Nat. Med. (N.Y.) 9, 796 (2003).

12. S.-J. Lin, C.-Y. Hsiao, Y. Sun, W. Lo, W.-C. Lin, G.-J. Jan, S.-H. Jee, and C.-Y. Dong, Opt. Lett. 30, 622 (2005).

13. Y. Sun, J. Su, W. Lo, S. Lin, S. Jee, and C. Dong, Opt. Express 11, 3377 (2003). 